\title{
Prolonged Infusion of Low Dose Gemcitabine in Advanced Pancreatic Carcinoma
}

\author{
Elsayed Mostafa Ali ${ }^{1}$, Ahmed M. Maklad ${ }^{1}$, Salah M. Khallaf ${ }^{*}$ \\ ${ }^{1}$ Clinical Oncology and Nuclear Medicine Department, Faculty of Medicine, Sohag, Egypt \\ ${ }^{2}$ Medical Oncology Department, South Egypt Cancer Institute, Assiut, Egypt \\ Email: *salahmab76@yahoo.com
}

How to cite this paper: Ali, E.M., Maklad, A.M. and Khallaf, S.M. (2020) Prolonged Infusion of Low Dose Gemcitabine in Advanced Pancreatic Carcinoma. Journal of Cancer Therapy, 11, 401-409.

https://doi.org/10.4236/jct.2020.117035

Received: March 13, 2020

Accepted: July 13, 2020

Published: July 16, 2020

Copyright $\odot 2020$ by author(s) and Scientific Research Publishing Inc. This work is licensed under the Creative Commons Attribution International License (CC BY 4.0).

http://creativecommons.org/licenses/by/4.0/

\begin{abstract}
Background: Gemcitabine was established as a monotherapy or in combination for locally advanced or metastatic pancreatic carcinoma. Aim: This study aimed to evaluate the efficacy of the low-dose gemcitabine over 6-hour infusion in patients with advanced pancreatic adenocarcinoma. Methods: 26 patients with locally advanced or metastatic pancreatic carcinoma were recruited into the study from December 2013 to October 2014. Patients received the treatment in Clinical Oncology Department, Sohag University, and Medical Oncology Department, Assiut University. Patients received low-dose gemcitabine $\left(250 \mathrm{mg} / \mathrm{m}^{2}\right)$ over 6-hour infusion, weekly for seven weeks and then on days 1 and 8 every 3 weeks till unacceptable toxicity or progression of the disease. Results: Twenty-six patients were enrolled in this study. After starting 7 weeks of treatment, the disease control rate was $38.5 \%$ in the form of complete response in $3.8 \%$ of patients, partial response in $26.9 \%$, and stationary response in $7.7 \%$. However, disease progression occurred in $61.5 \%$. Progression-free survivals were $65.38 \%, 23.07 \%, 7.69 \%$ and $3.84 \%$ after 3,6 , 9 and 12 months, respectively. Also, overall survivals at 3-month, 6-month, 9 -month, and 12 -month were $61.53 \%, 42.30 \%, 23.07 \%$, and $7.69 \%$, respectively. Conclusion: Prolonged infusion of low dose gemcitabine is a tolerable and a good option in locally advanced or metastatic pancreatic carcinoma. There may be a benefit of that protocol in patients with bad performance status. More clinical trials with a combination of other cytotoxic agents or target therapy are needed to get better survival and lesser toxicity.
\end{abstract}

\section{Keywords}

Gemcitabine, Pancreatic Cancer, Intravenous Infusion, Low-Dose

\section{Introduction}

Incidence of pancreatic carcinoma increased in USA, possibly because of in- 
creasing obesity, aging, and other causes [1]. Pancreatic cancer is the fourth most common mortality cancer related death among cancer patients [2]. Most patients who develop pancreatic adenocarcinoma, live less than 12 months with the length of patient survival greatly affected by disease stage at presentation, although few other markers of survival have been well characterized [3] [4]. Gemcitabine is an important treatment with 5-FU in locally advanced or metastatic pancreatic carcinoma; NCCN 2018 panel recommends gemcitabine as one option for $1^{\text {st }}$ line therapy [5] [6]. Continuous infusion of gemcitabine provided longer median survival compared with standard 30 minutes infusion, but with increased the risk of hematological toxicities [7]. In phase II study performed by Khaled et al.; 2008 in Egypt, 6 hours infusion of gemcitabine and cisplatin was an effective therapy for advanced cancer bladder; myelosuppression was mild. They concluded that the prolonged infusion of gemcitabine deserves to be tested in other malignancies [8]. There is no difference in survival between combination of gemcitabine with other cytotoxic agents and gemcitabine alone [9]. FOLFIRINOX regimen showed a survival benefit, but with increasing in toxicity, this option should be used for the treatment of patients with good performance status [10]. The study was performed to assess the outcome of patients with locally advanced or metastatic pancreatic carcinoma when treated with prolonged infusion of low dose gemcitabine.

\section{Methods}

During the period from December 2013 to October 2014, twenty-six patients with locally advanced or metastatic pancreatic carcinoma were recruited in this study from Clinical Oncology Department, Sohag University and Medical Oncology Department, Assiut University. Eligibility criteria included patients with 18 years or more, both sexes, performance status ECOG (0-2), with good organs functions. No Pregnancy or lactation was allowed during period of chemotherapy and fertile patients had to use effective contraception. We did pretreatment evaluation included history, clinical examination, laboratory tests (complete blood counts, kidney function, liver function, tumor marker CA19-9), and radiological studies (abdominal ultrasonography, chest x-ray and multi-slice computed tomography (CT) of the abdomen and pelvis). After complete work up, patients were clinically staged before treatment according to TNM clinical staging system.

Treatment plan consisted of continuous infusion of gemcitabine at a dose of $250 \mathrm{mg} / \mathrm{m}^{2}$ over 6 hours infusion given in weekly for seven weeks and then on day 1 and day 8 every 3 weeks until there is disease progression or unacceptable toxicity. Doses were adjusted according to blood counts, withheld if: Absolute neutrophilic count is $<1500 /$ ul or platelets count $<75,000 /$ ul on day 1 or if creatinine level $>1$ time $(x)$ upper normal limit (UNL) or aspartate aminotransferase $>5 \times$ UNL or bilirubin level $>1.5 \times$ UNL. In this case, laboratory investigations were repeated weakly and chemotherapy was restarted again as soon as counts allowed, with at least $50 \%$ of the dose to be administered. The patients were 
followed up by history, physical examination, $\mathrm{CBC}$, chemistry, liver function test every cycle. After 7 weeks of continuous infusion of gemcitabine, patients were followed by multi-slice CT of chest \& pelvi-abdominal and tumor marker CA 19-9. If there was radiological or biochemical response (partially or stationary), treatment was continued as day 1 and day 8 every 3 weeks then assessment by CT of chest \& pelvi-abdominal and tumor marker CA 19-9 every three cycles. If there was radiological or biochemical progression during treatment, we shifted to second line of chemotherapy (combination chemotherapy). We classified our patients according their demographics (age, gender, body mass index and performance status) and tumor characteristics (tumor site, tumor size, tumor grade, metastatic status, and CA 19-9 level).

\section{Statistical methods of analysis:}

Data collected and analyzed by computer program SPSS "ver. 21". The primary end-points were progression-free survival (PFS), which was defined as the time from the start of treatment to disease progression or death from any cause, whichever came first. Secondary end-points were overall survival (OS) and disease control rate (DCR). OS was defined as the time from the start of treatment to date of death from any cause, or date of last follow up, whichever came first. Disease control rate (DCR) was defined as sum of rates of stable disease (SD), complete response (CR), and partial response (PR). Univariate analysis was used through the presentation of continuous variables as median and range. Categorical variables are presented as frequency and percentage. Bivariate analysis was done to compare categorical variables using Chi-Square test or Fisher Exact test when appropriate. Kaplan-Meier method was used to estimate the survival time distribution and the median survival. The treatment difference between two groups was assessed by a log-rank test. A P-value less than 0.05 is considered as a cut-off.

\section{Results}

From December 2013, to October 2014, we enrolled 26 patients treated in Clinical Oncology Department, Sohag University and Medical Oncology Department, South Egypt Cancer Institute, Assiut University. Overall, 19 patients were male (73.1\%), and 7 patients were female (26.9\%) with a median age of 57.6 years (range 19 - 81). Forty-two percent $(42.3 \%)$ of patients were nonsmokers. Their main complains were abdominal pain (100\%) then jaundice (80.76\%) (Table 1).

As regard disease characteristics, pathological diagnosis of $\mathrm{T} 3$ and $\mathrm{T} 4$ were $57.6 \%$ and $15.4 \%$; respectively. Twenty-one of patients $(80.76 \%)$ had tumor at the head of pancreas, three patients (11.5\%) at the tail, two patients $(7.7 \%)$ at the body of pancreas. Eleven patients (42.3\%) were stented to relieve the obstructive jaundice. Sixty-five percent of patients had a solitary site of metastasis, which was the liver. While there were only five percent of patients had multiple sites of metastases, which included liver, lung, and bone (Table 1).

After treatment of 26 patients with continuous infusion of gemcitabine over 6-hour infusion weekly for seven weeks and then for day landday 8 every 3 weeks, there was disease control rate of $38.5 \%$ in the form of complete response 
in one patient $(3.8 \%)$, partial response in 7 patients $(26.9 \%)$ and stationary response in 2 patients $(7.7 \%)$, while there was a disease progression in 16 patients (61.5\%) (Figure 1).

Table 1. Demographics and baseline characteristics of enrolled patients $(n=29)$ receiving gemcitabine of low dose over 6-hour infusion.

\begin{tabular}{|c|c|c|}
\hline Characteristic & No & $\%$ \\
\hline \multicolumn{3}{|c|}{ Age, Years } \\
\hline Mean (Range) & \multicolumn{2}{|c|}{$57.69(19-81)$} \\
\hline \multicolumn{3}{|c|}{ Sex } \\
\hline Male & 19 & $73.1 \%$ \\
\hline Female & 7 & $26.9 \%$ \\
\hline \multicolumn{3}{|c|}{ Diabetes Mellitus } \\
\hline Type I & 2 & $7.7 \%$ \\
\hline Type II & 12 & $46.2 \%$ \\
\hline Non & 12 & $46.2 \%$ \\
\hline \multicolumn{3}{|c|}{ BMI } \\
\hline Obese & 1 & $3.8 \%$ \\
\hline Average & 7 & $26.9 \%$ \\
\hline Under built & 13 & $50.0 \%$ \\
\hline Cachectic & 5 & $19.2 \%$ \\
\hline \multicolumn{3}{|c|}{ ECOG PS } \\
\hline Grade 0 & 1 & $3.8 \%$ \\
\hline Grade I & 14 & $53.8 \%$ \\
\hline Grade II & 11 & $42.3 \%$ \\
\hline \multicolumn{3}{|c|}{ Clinical Manifestation } \\
\hline Abdominal pain & 26 & $100 \%$ \\
\hline Jaundice & 21 & $80.8 \%$ \\
\hline Pruritis & 3 & $11.5 .7 \%$ \\
\hline Nausea \& vomiting & 2 & $7.7 \%$ \\
\hline \multicolumn{3}{|c|}{ Pancreatic Tumor Site } \\
\hline Head & 21 & $80.76 \%$ \\
\hline Body & 2 & $7.7 \%$ \\
\hline Tail & 3 & $11.5 \%$ \\
\hline Multicentric & 0 & $0.0 \%$ \\
\hline \multicolumn{3}{|c|}{ Tumor Size } \\
\hline $\mathrm{T} 2$ & 7 & $26.9 \%$ \\
\hline $\mathrm{T} 3$ & 15 & $57.6 \%$ \\
\hline $\mathrm{T} 4$ & 4 & $15.4 \%$ \\
\hline \multicolumn{3}{|c|}{ Tumor Grade } \\
\hline Grade I/II & 17 & $65.4 \%$ \\
\hline Grade III & 6 & $23.1 \%$ \\
\hline Unknown & 3 & $11.5 \%$ \\
\hline \multicolumn{3}{|c|}{ Presence of Metastasis } \\
\hline Solitary & 17 & $65.4 \%$ \\
\hline Multiple & 5 & $19.2 \%$ \\
\hline Non & 4 & $15.4 \%$ \\
\hline \multicolumn{3}{|l|}{ CA19-9 level } \\
\hline Normal & 9 & $34.6 \%$ \\
\hline High & 17 & $65.4 \%$ \\
\hline
\end{tabular}

Abbreviations: BMI, Body Mass Index; ECOG PS, Eastern Cooperative Oncology Group Performance Status. 


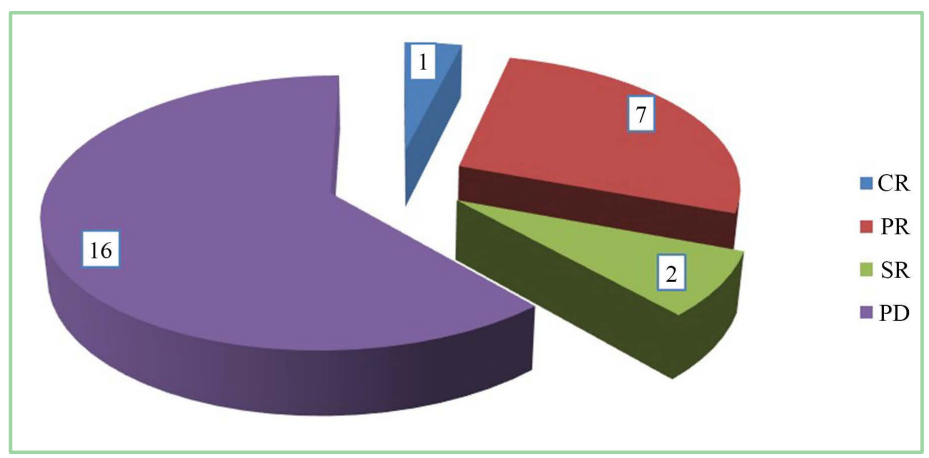

Figure 1. Pia chart of response rates in study group. CR, complete remission; $\mathrm{PD}$, progressive disease; $\mathrm{PR}$, partial remission; $\mathrm{SR}$, stationary response.

Hematological toxicity was the most significant adverse effect. Grade 3 and 4 neutropenia were observed in $8(36.7 \%)$ and $4(15.4 \%)$ patients, respectively. Grade 2,3 , and 4 anemia occurred in 9 (34.6\%), 13 (50\%), and 2 (7.7\%) patients, respectively. Grade 2 thrombocytopenia occurred only in 2 (7.7\%) patients. Also, non-hematological toxicity was observed such as increased creatinine level in 6 (23.0\%) patients, nausea occurred in all patients (100\%), vomiting grade 1, 2, and 3 experienced in 4 (15.3\%), 12 (46.1\%), 5(19.2\%) patients; respectively. Diarrhea grade 1 and 2 are observed in 12 (46.1\%), 4 (15.3\%) patients; respectively. Six (23\%) patients experienced alopecia (Table 2).

Mean progression-free survival (PFS) was 3.65 months, 95\% CI $2.34-4.95$ months (Figure 2). Progression-free survival at 3-month, 6-month, 9-month, and 12 -month were $65.38 \%, 23.07 \%, 7.69 \%$, and $3.84 \%$, respectively (Table 3 ). The mean overall survival (OS) was 5.69 months, 95\% CI 4.34 - 7.52 months (Figure 3). Overall survival at 3-month, 6-month, 9-month, and 12-month were $61.53 \%, 42.30 \%, 23.07 \%$, and $7.69 \%$, respectively (Table 3). Performance status was significantly correlated with PFS (P-value $<0.01$ ). While other factors which were theage, the tumor size, the tumor grade, the tumor marker CA19-9, body mass index, and smoking were not significant either for PFS or OS.

\section{Discussion}

In the present study, the median of age was 59 years (range 19 - 81) with peak incidence in $5^{\text {th }}$ decade and $6^{\text {th }}$ decade; 7 cases $(26.9 \%)$ per each, and that was comparable with series of Tempro et al., 2003 in which the median of age was 62 years and the peak of incidence was in $6^{\text {th }}$ decade [11]. Poplin et al. observed that peak incidence was in the fifth and sixth decades of life [12]. Regarding disease control rate, it was $38.5 \%$, which is comparable with study conducted by Poplin et al., in which response rate was $36 \%$.

The overall survival in this study was 5.69 months, 95\% CI 4.34 - 7.52 months and 12 -month survival was $7.69 \%$ and that was less than reported by Poplin et al. [12], in which single-agent gemcitabine (GEM) $1000 \mathrm{mg} / \mathrm{m}^{2}$ over 30 -minute infusion compared with fixed-dose rate (FDR) GEM $1500 \mathrm{mg} / \mathrm{m}^{2}$ over 150 -minutes and GEM $1000 \mathrm{mg} / \mathrm{m}^{2}$ over 100 -minutes infusion/day 1 plus oxaliplatin $100 \mathrm{mg} / \mathrm{m}^{2} /$ day 
2 every 14 days (GEMOX) . The median survival and 1-year survival were 4.9 months and 16\% for GEM, 6.2 months, and 21\% for GEM FDR (P=0.04), and 5.7 months and $21 \%$ for GEMOX $(P=0.22)$. This contrast may be due to different biological capacity of the patients or different treatment schedule and dosage. As patients with good performance status (PS 0,1$)$ were higher than those in our study (more than $80 \%$ of the patients in the three groups of Poplin's study versus $57.6 \%$ in our study.

Table 2. Toxicities according to CTCAE (version 4.03) in 26 patients.

\begin{tabular}{ccccc}
\hline & Grade 1 & Grade 2 & Grade 3 & Grade 4 \\
\hline $\begin{array}{c}\text { Hematological } \\
\text { Neutropenia }\end{array}$ & - & - & $8(30.7 \%)$ & $4(15.3 \%)$ \\
Anemia & - & $9(34.6 \%)$ & $13(50 \%)$ & $2(7.7 \%)$ \\
Thrombocytopenia & $5(19.2 \%)$ & $2(7.7 \%)$ & - & - \\
Non-Hematological & $2(7.7 \%)$ & $4(15.3 \%)$ & - & - \\
Creatinine & $6(23.0 \%)$ & $14(53.8 \%)$ & $6(6.6 \%)$ & - \\
Nausea & $4(15.3 \%)$ & $12(46.1 \%)$ & $5(19.2 \%)$ & - \\
Vomiting & $12(46.1 \%)$ & $4(15.3 \%)$ & - & - \\
Diarrhea & $6(23.0 \%)$ & - & - \\
Alopecia & & & & - \\
\hline
\end{tabular}

Abbreviation: CTCAE, the Common Terminology Criteria for Adverse Events.

Table 3. Distribution of Progression-Free Survival (DFS) and Overall Survival (OS) of all patients in the follow up period (12 months), presented as number of patients and their percent.

\begin{tabular}{ccccc}
\hline Survival category & 3 months & 6 months & 9 months & 12 months \\
\hline PFS & $17(65.38 \%)$ & $6(23.07 \%)$ & $2(7.69 \%)$ & $1(3.84 \%)$ \\
OS & $16(61.53 \%)$ & $11(42.3 \%)$ & $6(23.07 \%)$ & $2(7.69 \%)$ \\
\hline
\end{tabular}

Abbreviations: OS, Overall Survival; Progression-Free Survival (PFS).

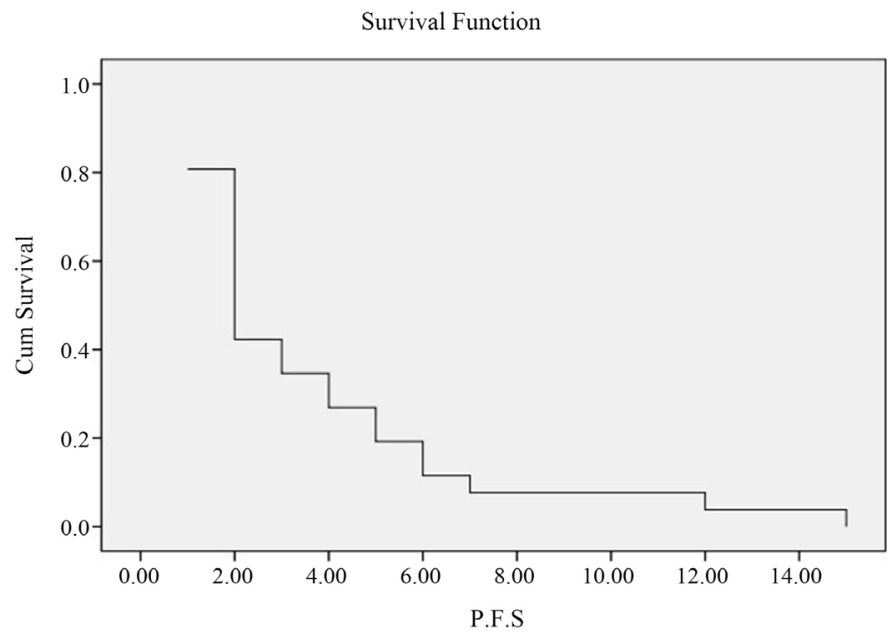

Figure 2. Kaplan-Meier plot of progression-free survival. 


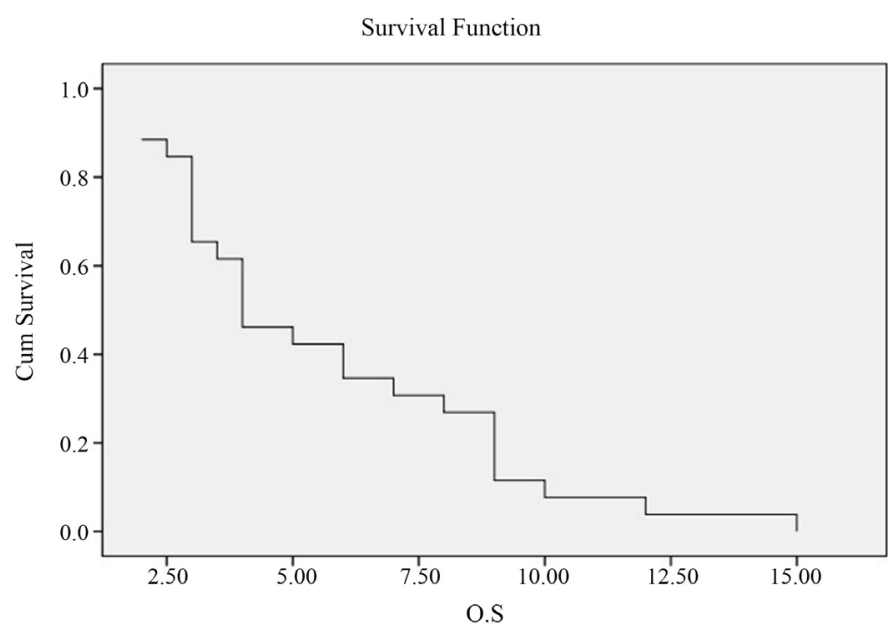

Figure 3. Kaplan-Meier plot of overall survival.

The treatment was subjectively well tolerated in the present study, although hematological toxicity was the most significant adverse effect. Grade 3, 4 neutropenia observed in 8 (36.7\%), 4 (15.4\%) patients respectively. Grade 3 and 4 anemia occurred in $13(50 \%)$ and $2(7.7 \%)$ patients; respectively, while grade 1 and 2 thrombocytopenia occurred in $5(19.2 \%)$ and 2 (7.7\%) patients; respectively. These incidence rates were less than that of Tempro et al. [11] in which grade 3/4 thrombocytopenia, grade 3/4neutropenia, and grade 4 anemia were observed in FDR arm as follows: 37\%, 48.8\%, 9.3\%; respectively.

Also, our incidence rates of toxicities were less than that reported by Poplin et al. [12] in which the most significant toxicity was myelosuppression, which was worse in GEM FDRarm. As they stated that grade 3/4 thrombocytopenia was $33 \%$, neutropenia $3 / 4$ was $59 \%$ and anemia $3 / 4$ was $19 \%$. This emphasizes that the low-dose gemcitabine protocol has a lower incidence of toxicity than that caused by standard dose protocol of gemcitabine.

The main limitation of our study is the small sample size, but we did larger randomized phase II study based on the result of this trial [13].

This study was presented as an abstract [14] — not a full paper-on the 2016 ESMO World Congress on Gastrointestinal Cancer was held in Barcelona, Spain; 29 June-2 July 2016. We want to share our practice in this study by publishing it as a full text to be more useful.

\section{Conclusion}

Prolonged infusion of low dose gemcitabine is a tolerable and a good option in locally advanced or metastatic pancreatic carcinoma. There may be a benefit of that protocol, especially patients with bad performance status. More clinical trials with a combination of other cytotoxic agents or target therapy are needed to get better survival and lesser toxicity.

\section{Conflicts of Interest}

The authors declare no conflicts of interest regarding the publication of this paper. 


\section{References}

[1] Simard, E.P., Ward, E.M., Siegel, R. and Jemal, A. (2012) Cancers with Increasing Incidence Trends in the United States: 1999 through 2008. CA: A Cancer Journal for Clinicians, 62, 118-128. https://doi.org/10.3322/caac.20141

[2] Siegel, R.L., Miller, K.D. and Jemal, A. (2017) Cancer Statistics. CA: A Cancer Journal for Clinicians, 67, 7-30. https://doi.org/10.3322/caac.21387

[3] Sant, M., Allemani, C., Santaquilani, M., Knijn, A., Marchesi, F. and Capocaccia, R. (2009) EUROCARE-4: Survival of Cancer Patients Diagnosed in 1995-1999: Results and Commentary. European Journal of Cancer, 45, 931-991. https://doi.org/10.1016/j.ejca.2008.11.018

[4] Hidalgo, M. (2010) Pancreatic Cancer. The New England Journal of Medicine, 362, 1605-1617. https://doi.org/10.1056/NEJMra0901557

[5] Burris III, H.A., Moore, M.J., Andersen, J., et al. (1997) Improvements in Survival and Clinical Benefit with Gemcitabine as First-Line Therapy for Patients with Advanced Pancreas Cancer: A Randomized Trial. Journal of Clinical Oncology, 15, 2403-2413. https://doi.org/10.1200/JCO.1997.15.6.2403

[6] Tempero, M.A., Malafa, M.P., Al-Hawary, M., Asbun, H., Bain, A., et al. (2017) Pancreatic Adenocarcinoma, Version 2.2017: Clinical Practice Guidelines in Oncology. Journal of the National Comprehensive Cancer Network, 15, 1028-1061. https://doi.org/10.6004/jnccn.2017.0131

[7] Xie, J., Yuan, J. and Lu, L. (2014) Gemcitabine Fixed-Dose Rate Infusion for the Treatment of Pancreatic Carcinoma: A Meta-Analysis of Randomized Controlled Trials. Diagnostic Pathology, 9, 214. https://doi.org/10.1186/s13000-014-0214-8

[8] Emara, M.E., Gaafar, R.M., Mansour, O., Abdel Warith, A., Zaghloul, M.S. and El Malt, O. (2008) Primary Chemotherapy with Low-Dose Prolonged Infusion Gemcitabine and Cisplatin in Patients with Bladder Cancer: A Phase II Trial. Urologic Oncology, 26, 133-136. https://doi.org/10.1016/j.urolonc.2007.01.013

[9] Di Marco, M., Di Cicilia, R., Macchini, M., Nobili, E., Vecchiarelli, S., Brandi, G., et al. (2010) Metastatic Pancreatic Cancer: Is Gemcitabine Still the Best Standard Treatment? Oncology Reports, 23, 1183-1192. https://doi.org/10.3892/or_00000749

[10] Conroy, T., Desseigne, F., Ychou, M., Bouche, O., Guimbaud, R., Becouarn, Y., et al. (2011) FOLFIRINOX versus Gemcitabine for Metastatic Pancreatic Cancer. The New England Journal of Medicine, 364, 1817-1825. https://doi.org/10.1056/NEJMoa1011923

[11] Tempero, M., Plunkett, W., Ruiz Van Haperen, V., Hainsworth, J., Hochster, H., Lenzi, R., et al. (2003) Randomized Phase II Comparison of Dose-Intense Gemcitabine: Thirty-Minute Infusion and Fixed Dose Rate Infusion in Patients with Pancreatic Adenocarcinoma. Journal of Clinical Oncology, 21, 3402-3408. https://doi.org/10.1200/JCO.2003.09.140

[12] Poplin, E., Feng, Y., Berlin, J., Rothenberg, M.L., Hochster, H., Mitchell, E., et al. (2009) Phase III, Randomized Study of Gemcitabineand Oxaliplatin versus Gemcitabine (Fixed-Dose Rate Infusion) Compared with Gemcitabine (30-Minute Infusion) in Patients with Pancreatic Carcinoma E6201: A Trial of the Eastern Cooperative Oncology Group. Journal of Clinical Oncology, 27, 3778-3785. https://doi.org/10.1200/JCO.2008.20.9007

[13] Khallaf, S., Ali, E., Maklad, A., Samir, M., Amin, A., El-masry, M., Sayed, M. and Osama, D. (2020) In Frail Elderly Patients, Low-Dose Gemcitabine over 6-Hour Infusion Is Equally Effective and Less Toxic Than the Standard Gemcitabine Proto- 
col for Advanced Pancreatic Adenocarcinoma: A Randomized Phase II Trial. JCT, 11, 124-141. https://doi.org/10.4236/jct.2020.113011

[14] Ali, E.M., Maklad, A.M., Khallaf, S.M. and Samir, M.M. (2016) RM-029: Outcome of Continuous Infusion of Low Dose Gemcitabine in Locally Advanced and Metastatic Pancreatic Carcinoma. Annals of Oncology, 27, ii92.

https://doi.org/10.1093/annonc/mdw201.28

\section{List of Abbreviations}

5-FU: 5-Fluorouracil

BMI: Body Mass Index

CR: Complete Response

CT: Computed Tomography

CTCAE: Common Terminology Criteria for Adverse Events

DCR: Disease Control Rate

ECOG PS: Eastern Cooperative Oncology Group Performance Status

FDR: Fixed-Dose Rate

GEMOX: Gemcitabine plus Oxaliplatin

NCCN: National Comprehensive Cancer Network

OS: Overall Survival

PFS: Progression-Free Survival

PR: Partial Remission

PR: Partial Response

SD: Stable Disease $=$ SR: Stationary Response

TNM: Tumor (T), Nodes (N), and Metastases (M)

UNL: Upper Normal Limit 